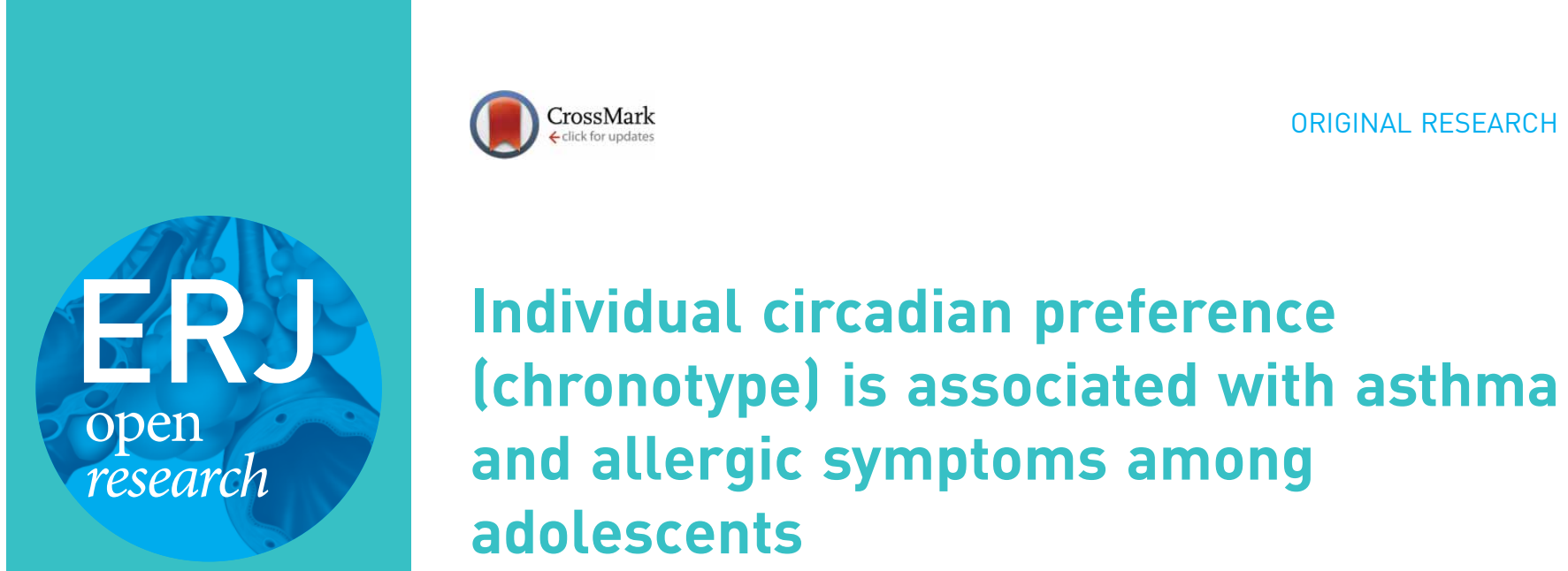

To the Editor:

Circadian rhythm is an endogenously driven cyclical process that regulates most of our bodily functions, such as sleep-wakefulness cycle, metabolism, and a wide range of cellular, molecular and behavioural processes. Chronotype, or an individual's preferred time of sleep and activity, is one of the major internal cues that helps to harmonise one's biological events according to its circadian timing [1]. Three major variations of chronotype exist among humans, those who prefer to wake up early and sleep early at night (known as morning types or "larks"), those who prefer late sleep at night and late waking up in the morning (referred to as evening type or "owls") and those who remain in between (intermediate type) [1].

An increasing body of evidence have shown that chronotype difference, for example, those with more preference to night-time activities, is associated with higher risks of cardiac, metabolic and psychological disorders [2-4]. In recent years, some studies have been published stating the association between respiratory diseases and circadian rhythm [5-8]. However, little is known about the plausible association between chronotype and asthma/allergic diseases. In this paper, we aimed to explore the relationship between chronotype and allergy-related disorders among adolescents.

We analysed data on 1684 Indian adolescents (aged between 13 and 14 years) from the Prevalence and Risk Factors of Asthma and Allergy-Related Diseases among Adolescents (PERFORMANCE) study [9]. The study was approved by the Clinical Research Ethics Committee of Allergy \& Asthma Research Centre, Kolkata (CREC-AARC-04/2016).

The details of the study design, methodologies and ethical concerns have been described elsewhere [9]. Briefly, we administered an English-back Bengali translated version of the International Study of Asthma and Allergy in Childhood (ISAAC) Phase III questionnaire among students of the specified age group of 16 randomly selected schools of West Bengal to obtain information about respiratory symptoms (namely wheeze, rhinitis, rhinoconjunctivitis and asthma). Each respiratory symptom was defined using both "ever" for a positive response to the question "have you ever had that symptom?" (e.g. wheeze) and "current" if the participant also reported having that symptom within the last 12 months. Additionally, demographic and social characteristics of the participant and information about the neighbourhood environment were also collected by questionnaire. Asthma was assessed from the questionnaire. Chronotypes of the participants (categorised into morning, evening and intermediate types) were determined using a validated Bengali version of the reduced Morningness-Eveningness Questionnaire (rMEQ) [10].

The associations between each respiratory symptom (wheeze, rhinitis, rhinoconjunctivitis and asthma) and individual chronotype (morning type as the reference category) were assessed, overall and by sex, using modified Poisson regression models [11], adjusting for sex, area of residence (rural, industrial suburban, nonindustrial suburban and urban), parental atopy/asthma (yes/no), exposure to second-hand smoke

@ERSpublications

Individual preference of sleep and activity (chronotype) might play an important role on asthma and allergic diseases in adolescents: this link underscores a potential plausible behavioural intervention for asthma and allergic diseases in young population https://bit.ly/2THta9I

Cite this article as: Haldar P, Carsin A-E, Debnath S, et al. Individual circadian preference (chronotype) is associated with asthma and allergic symptoms among adolescents. ERJ Open Res 2020; 6: 00226-2020 [https://doi.org/10.1183/23120541.00226-2020].

Copyright $\odot$ ERS 2020. This article is open access and distributed under the terms of the Creative Commons Attribution NonCommercial Licence 4.0. 
(SHS) at home (yes/no), exposure to pets (yes/no) and number of siblings. As an exploratory analysis, we checked for effect modification by stratifying by 1) parental atopy/asthma, 2) exposure to SHS at home, 3) exposure to pet at home and 4) number of siblings.

$42 \%$ of the participants were girls. $42 \%$ and $9 \%$ of the participants were identified as morning and evening types, respectively, while the rest (49\%) were intermediate types. $13 \%$ of the participants had ever wheeze, while ever rhinitis and asthma were present among $21 \%$ and $11 \%$ participants, respectively. Current wheeze, rhinitis and allergic rhinitis (rhinoconjunctivitis) were present among 7\%, 17\% and $9 \%$ participants, respectively. Based on the residential domicile, $42 \%$ of participants lived in rural areas, $25 \%$ in urban areas and $33 \%$ in suburbs (17\% in industrial suburbs and $16 \%$ in nonindustrial suburbs). Parental atopy/asthma was reported by $18 \%$ of participants. $38 \%$ reported to have an exposure to SHS at home and $45 \%$ of the participants had an exposure to pet at home. $82 \%$ had one or more siblings.

Compared to the morning-type individuals, those that were evening type had higher risk of current (but not ever) wheeze (6.4\% versus $11.7 \%$; relative risk (RR) $1.85,95 \%$ CI 1.02-3.35) (table 1). A similar result was observed for participants reporting ever or current rhinitis, but additionally, those that were intermediate type also demonstrated significantly higher risks than the morning type. Rhinoconjunctivitis was also higher in the intermediate-type (RR 1.51, 95\% CI 1.02-2.23) and evening-type groups (RR 2.76, 95\% CI 1.94-3.92) compared to those in the morning-type group. RR for asthma was three times higher among the evening-type participants (23.6\% versus $6.2 \%$; RR 2.67, 95\% CI 1.65-4.31) than the morning types. No difference was observed for the association between chronotype and the symptoms after stratification by sex. We did not find evidence for effect modification by parental atopy/asthma, exposure to SHS at home, exposure to pet at home and number of siblings. In this study, we found that chronotype was associated with respiratory symptoms among adolescents. Evening-type adolescents had consistently more respiratory symptoms than the morning types. This effect was not restricted to the evening types but was also observed, to a smaller degree, among the intermediate types. We also found that the association was not modified by the presence of potential interpersonal, environmental or genetic factors.

Asthma and allergic diseases are known to worsen at night; however, the pathophysiology of asthma and allergic diseases is diverse, and may potentially be linked to an individual's preference to rest and activity.

TABLE 1 Association between chronotype and respiratory symptoms

Unadjusted RR $(95 \% \mathrm{Cl})$

\section{Ever wheeze}

Morning type

Intermediate type

Evening type

Current wheeze

Morning type

Intermediate type

Evening type

Ever rhinitis

Morning type

Intermediate type

Evening type

Current rhinitis

Morning type

Intermediate type

Evening type

Current rhinoconjunctivitis

Morning type

Intermediate type

Evening type

Ever asthma

Morning type

Intermediate type

Evening type
$0.98(0.75-1.28)$

1

$1.03(0.70-1.51)$

$1.83(1.08-3.11)$

1

$1.69(1.36-2.11)$

$2.47(1.86-3.28)$

1

$1.87(1.45-2.43)$

2.97 (2.14-4.11)

$1.59(1.10-2.29)$

2.65 (1.65-4.28)

1

2.15 (1.53-3.03)

3.79 (2.49-5.75)
1.50 (1.02-2.21)
Adjusted RR $(95 \% \mathrm{CI})$

Each respiratory symptom was tested in a single model. In adjusted models, sex, area of residence, parental atopy/asthma, exposure to second-hand smoke at home, having a pet at home and number of siblings were included as confounders. RR: relative risk. Bold type indicates $p<0.05$. 
Two recent reports examined the circadian rhythmicity of airway eosinophils, fractional exhaled nitric oxide and volatile organic compounds in the exhaled breath of the patients with asthma $[7,8]$, although the underlying mechanism is still not well understood. It has been proposed that a long evening hour may impair sleep and melatonin homeostasis, while others proposed a longer circadian period and greater sleep debt among the evening-type individuals [12]. It is already known that evening-types are easily more prone to circadian misalignments that could eventually lead to circadian clock dysfunction which triggers several down-stream mechanisms including altered immune systems in the lungs [13].

It can also be assumed that evening-type individuals are more exposed to artificial light at night, an environmental hazard [2] and known suppressor of melatonin. Melatonin, the sleep hormone, has recently been studied for its immunomodulatory role, and has turned out to be a potential player in asthma and allergic diseases, especially when the biological clock is dysregulated or disrupted [13].

Nowadays, lifestyle and behavioural factors are known to be potentially linked with asthma morbidity [14]. Our findings of the role of individual preference to sleep and activity on the risk of allergy and asthma describe the importance of assessing circadian typology as a plausible factor for the disease, thus would help clinicians and researchers to pay attention to this lesser-known pathway of asthma and allergic diseases.

This is the first study to report on the possible association between chronotype and respiratory symptoms among adolescents. Our results are concordant with the findings in adults of the only available published report on this topic [15]. However, our findings have some limitations. Chronotype assessment was based on the questionnaire response and we could not measure the duration of night-time activity, rest and activity cycle, and sleep duration which could have better reflected the degree of circadian desynchronisation. Secondly, a very small proportion of our study population (only 9\%) was evening type. Similar studies in other populations with relatively higher proportions of evening types might be useful to substantiate the robustness of our results. Thirdly, any clinical measurement such as lung function testing, blood cell count or allergen-sensitivity test was beyond the scope of this observational study. Finally, this is a cross-sectional study; therefore, we cannot draw any causal inference between chronotype and respiratory symptoms.

In conclusion, we demonstrate an association between chronotype and respiratory health; this new finding underscores the importance of understanding this exigent interpersonal attribute as an important clinical correlate of respiratory health, thus warranting more research in the field of respiratory chronomedicine to comprehend the mechanisms behind those associations.

\section{Prasun Haldar ${ }^{1,10}$, Anne-Elie Carsin ${ }^{2,3,4,10}$, Smriti Debnath ${ }^{5}$, Santi Gopal Maity ${ }^{6}$, Isabella Annesi-Maesano ${ }^{7}$, Judith Garcia-Aymerich $\circledast^{2,3,4}$, Arghya Bandyopadhayay ${ }^{8}$, Saibal Moitra $\oplus^{5,9}$, Manolis Kogevinas ${ }^{2,3,4}$ and Subhabrata Moitra $\oplus^{2,3,4}$ on behalf of the PERFORMANCE Study Group}

${ }^{1}$ Dept of Biological Sciences, Midnapore City College, Midnapore, India. ${ }^{2}$ ISGlobal, Barcelona, Spain. ${ }^{3}$ Universitat Pompeu Fabra, Barcelona Spain. ${ }^{4}$ CIBER Epidemiología y Salud Pública, Barcelona, Spain. ${ }^{5}$ Dept of Pneumology, Allergy and Asthma Research Centre, Kolkata, India. ${ }^{6}$ Dept of Physiology, Uluberia College, Uluberia, India. ${ }^{7}$ IPLESP INSERM and Sorbonne Université, Paris, France. ${ }^{8}$ Indian Institute of Science Education and Research, Kolkata, India. ${ }^{9}$ Dept of Allergy and Immunology, Apollo Gleneagles Hospitals, Kolkata, India. ${ }^{10}$ These authors contributed equally.

Correspondence: Subhabrata Moitra, Alberta Respiratory Centre, Division of Pulmonary Medicine, Dept of Medicine, 559 HMRC, University of Alberta, Edmonton, AB T6G 2S2, Canada. E-mail: moitra@ualberta.ca

Received: 28 April 2020 | Accepted after revision: 17 May 2020

Acknowledgement: The authors are grateful to the administrations of the attended schools for allowing to conduct the study, the students for participating in this study and the parents for their consent. Part of the study's results were presented in the 30th Conference of the International Society for Chronobiology, held in Warsaw, Poland, on 2-5 July 2019.

Data sharing policy: Our data contain sensitive personal information of the participants and may potentially disclose their identity. However, an anonymous dataset can be shared upon considerable request made to the corresponding author.

The PERFORMANCE collaborators: Anne-Elie Carsin (ISGlobal, Universitat Pompeu Fabra (UPF) and CIBER Epidemiología y Salud Pública (CIBERESP), Barcelona, Spain), Arghya Bandyopadhyay (Department of Chemical Sciences, IISER-Kolkata, Kolkata, India), Atanu Ghosh (Dept of Statistics, Presidency University, Kolkata, India), Dwiptirtha Chattopadhyay (North Bengal St Xavier's College, Rajganj, India), Isabella Annesi-Maesano (INSERM and 
Sorbonne Université, Paris, France), Judith Garcia-Aymerich (ISGlobal, UPF and CIBERESP, Barcelona, Spain), Manolis Kogevinas (ISGlobal, UPF and CIBERESP, Barcelona, Spain), Moumita Biswas (Santipur State General Hospital, Santipur, India), Ritabrata Mitra (Dept of Pulmonary Medicine, Institute of Post Graduate Medical Education and Research and Seth Sukhlal Karnani Memorial Hospital, Kolkata, India), Saibal Moitra (Dept of Pneumology, Allergy and Asthma Research Centre, Kolkata, India), Santi Gopal Maity (Dept of Physiology, Uluberia College, Uluberia, India), Soumya Bhattacharjee (Dept of Chest Medicine, Murshidabad Medical College and Hospital, Berhampore, India), Soumya Sengupta (Charnock Hospital, Kolkata, India), Subhabrata Moitra (Alberta Respiratory Centre, Dept of Medicine, University of Alberta, Edmonton, Canada), Sujoy Saha (BR Singh Hospital, Kolkata, India) and Tarunendu Mapder (Division of Clinical Pharmacology, Dept of Medicine, Indiana University School of Medicine, Indianapolis, IN, USA).

Author contributions: P. Haldar, I. Annesi-Maesano, J. Garcia-Aymerich, Saibal Moitra and Subhabrata Moitra conceived the study design; P. Haldar, S. Debnath, S.G. Maity and Subhabrata Moitra collected the data; A-E. Carsin, M. Kogenvinas and Subhabrata Moitra analysed and interpreted the data; P. Haldar, M. Kogevinas and Subhabrata Moitra wrote the manuscript; all other authors revised the manuscript and provided intellectual contents. P. Haldar and Subhabrata Moitra have full access to the data and take the responsibility for the accuracy and authenticity of the data.

Conflict of interest: P. Haldar has nothing to disclose. A-E. Carsin has nothing to disclose. S. Debnath has nothing to disclose. S.G. Maity has nothing to disclose. I. Annesi-Maesano has nothing to disclose. J. Garcia-Aymerich has nothing to disclose. A. Bandyopadhyay has nothing to disclose. Saibal Moitra has nothing to disclose. M. Kogevinas has nothing to disclose. Subhabrata Moitra reports a Young Scientist sponsorship and a long-term fellowship from the European Respiratory Society, and nonfinancial support from Oxford Publications, outside the submitted work.

Support statement: This study is a part of the Prevalence and Risk Factors of Asthma and Allergy-Related Diseases among Adolescents (PERFORMANCE) Study. The main study is supported by the internal research support facility of Allergy \& Asthma Research Centre, Kolkata, India.

\section{References}

1 Adan A, Archer SN, Hidalgo MP, et al. Circadian typology: a comprehensive review. Chronobiol Int 2012; 29: $1153-1175$.

2 West AC, Smith L, Ray DW, et al. Misalignment with the external light environment drives metabolic and cardiac dysfunction. Nat Commun 2017; 8: 417.

$3 \mathrm{Yu} \mathrm{JH}$, Yun $\mathrm{CH}, \mathrm{Ahn} \mathrm{JH}$, et al. Evening chronotype is associated with metabolic disorders and body composition in middle-aged adults. J Clin Endocrinol Metab 2015; 100: 1494-1502.

4 Antypa N, Verkuil B, Molendijk M, et al. Associations between chronotypes and psychological vulnerability factors of depression. Chronobiol Int 2017; 34: 1125-1135.

5 Sundar IK, Yao H, Sellix MT, et al. Circadian molecular clock in lung pathophysiology. Am J Physiol Lung Cell Mol Physiol 2015; 309: L1056-L1075.

6 Truong KK, Lam MT, Grandner MA, et al. Timing matters: circadian rhythm in sepsis, obstructive lung disease, obstructive sleep apnea, and cancer. Ann Am Thorac Soc 2016; 13: 1144-1154.

7 Durrington HJ, Gioan-Tavernier GO, Maidstone RJ, et al. Time of day affects eosinophil biomarkers in asthma: implications for diagnosis and treatment. Am J Respir Crit Care Med 2018; 198: 1578-1581.

8 Wilkinson M, Maidstone R, Loudon A, et al. Circadian rhythm of exhaled biomarkers in health and asthma. Eur Respir J 2019; 54: 1901068.

9 Bhattacharjee S, Haldar P, Gopal Maity S, et al. Prevalence and risk factors of asthma and allergy-related diseases among adolescents (PERFORMANCE) study: rationale and methods. ERJ Open Res 2018; 4: 00034-2018.

10 Haldar P, Bhattacharjee S, Maity SG, et al. Chronotype assessment of the Bengalese adolescents: an observational study using a Bengali version of the reduced morningness-eveningness questionnaire (rMEQ). Biol Rhythm Res 2019; [https://doi.org/10.1080/09291016.2019.1571702].

11 Zhou G. A modified Poisson regression approach to prospective studies with binary data. Am J Epidemiol 2004 159: 702-706.

12 Duffy JF, Rimmer DW, Czeisler CA. Association of intrinsic circadian period with morningness-eveningness, usual wake time, and circadian phase. Behav Neurosci 2001; 115: 895-899.

13 Marseglia L, D’Angelo G, Manti S, et al. Melatonin and atopy: role in atopic dermatitis and asthma. Int J Mol Sci 2014; 15: 13482-13493.

14 Oland AA, Booster GD, Bender BG. Psychological and lifestyle risk factors for asthma exacerbations and morbidity in children. World Allergy Organ J 2017; 10: 35.

15 Merikanto I, Englund A, Kronholm E, et al. Evening chronotypes have the increased odds for bronchial asthma and nocturnal asthma. Chronobiol Int 2014; 31: 95-101. 